\title{
Análise cinemática da variabilidade do membro de suporte dominante e não dominante durante o chute no futsal
}

\author{
Fabio A. Barbieri \\ Paulo R. Santiago \\ Lilian T. Gobbi \\ Sergio A. Cunha
}

\author{
Departamento de Educação Física - IB - UNESP \\ Rio Claro \\ Brasil
}

\section{RESUMO}

O objetivo do estudo foi analisar o desempenho e a variabilidade do movimento da articulação do quadril, joelho e tornozelo do membro de suporte do chute realizado com o membro dominante e não dominante no futsal. Doze participantes executaram cinco chutes com cada membro realizados com o dorso do pé com máxima velocidade e objetivo de acertar um alvo fixo. Os movimentos do membro de suporte foram filmados, sendo as imagens transferidas para o computador e os dados tridimensionais obtidos através do software Dvideow. Para a análise da variabilidade do movimento foram calculados os autovalores das matrizes de covariância das articulações do quadril, joelho e tornozelo do membro dominante e não dominante para cada instante de tempo, sendo verificada diferença entre os lados através da razão entre a raiz quadrada dos autovalores das articulações, sendo utilizada a função distribuição acumulada. Para o desempenho foi observado o local de acerto da bola após os chutes. Os resultados mostraram menor variabilidade das articulações do joelho e do tornozelo do membro dominante em alguns momentos do ciclo de chute em relação às respectivas articulações não dominantes e melhor desempenho do membro dominante, concluindo que o desempenho é influenciado pela variabilidade.

Palavras-chave: biomecânica, variabilidade do movimento, assimetria, desempenho

\begin{abstract}
Dominant and non-dominant support limb kinematics variability during futsal kick

The purpose of this study was to examine the performance and hip, knee and ankle movement variability of the support limb in the kick performed with the dominant and non-dominant limb in futsal. Twelve skilled male participants performed five maximal instep kicks with each limb and aimed to hit a fixed target. Their movements were recorded and the images were transferred to a computer, and analysis of the 3D data was made by Dvideow software. The Eigen values of the covariance matrix of the hip, knee and ankle joints of the dominant and non-dominant limb for each time instant were calculated to analyze movement variability. The ratio between the square roots was calculated for each time instant and the cumulative distribution function was used to verify the difference between the sides. The location of the ball related to the target after the kicks was measured for the performance values. The results presented less variability of the knee and ankle joints of the dominant limb at some moments of the kick cycle in comparison to the respective non-dominant joints. The dominant limb presented a better performance and we therefore concluded that performance is influenced by variability.
\end{abstract}

Key-words: biomechanics, movement variability, asymmetry, performance 


\section{INTRODUÇÃO}

Na prática do futsal é muito importante que o atleta utilize tanto o membro dominante (MD) quanto o não dominante (MND) durante a partida. O membro preferido ou dominante é aquele que apresenta melhor desempenho nas acções ${ }^{(23,26)}$, sendo que a preferência pedal é definida de acordo com o papel do membro na tarefa $(13,26)$. Desta forma, para as pessoas ditas destras o membro esquerdo é dominante para o suporte e estabilização do corpo - membro de suporte ${ }^{(12)}$ - enquanto que o membro direito é o não dominante, sendo o inverso para o membro de chute que é quem tem o contacto com a bola.

Com isso, para que o atleta tenha óptima eficiência durante a prática do futsal é interessante que este desenvolva desempenho semelhante entre os membros homólogos. Assim, ele conseguirá realizar muito bem suas ações técnicas e também suas funções tácticas, não ficando limitado a certos movimentos ou a determinados locais da quadra.

Por isso, a ambidestria é um factor significante para o rendimento do atleta de futsal. As equipes que possuem atletas ambidestros levam vantagens na técnica e na táctica por causa da grande versatilidade e coordenação dos jogadores durante a partida(30) e também por esses jogadores apresentarem maior proficiência que o esportista que possuem um membro como dominante devido à natureza do futsal(24), levando vantagens durante o jogo por utilizar estratégias diferenciadas $(30,8)$.

Apesar disso, poucos atletas desenvolvem simetria de movimento e desempenho entre os membros inferiores, evidenciada quando o movimento de chute é analisado(8). A diferenciação entre os lados nos movimentos de chute é palco de diversos estu$\operatorname{dos}(3,4,5,15,19,21,32)$. No entanto, existe uma predominância em trabalhos que analisam o membro que realiza o contacto com a bola. Todavia, este não é o único membro que participa desta acção, já que além dele também é utilizado o membro de suporte, mas a maioria dos trabalhos que analisam este membro durante o chute se restringe à verificação das variáveis biomecânicas do movimento e a força de reacção do solo sobre este membro(2, 17, 27).

Considerando que a maioria dos jogadores prioriza um dos membros para a realização dos movimentos e que a ambidestria auxilia no desempenho do fut- sal, é imprescindível analisar as diferenças de movimento entre os membros contra-laterais de suporte. Um dos factores preponderantes para a simetria de movimento entre os lados é a variabilidade. Uma grande variabilidade de movimento não é característica de uma acção habilidosa(4). Ao contrário, uma acção habilidosa requer consistência de movimento( ${ }^{(9,}$ 33), especialmente quando não há variação no ambiente. Vale lembrar que quanto maior for a consistência do movimento mais bem sucedida será a realização da tarefa. Logo, a consistência do movimento só é alcançada através da redução de variabilidade ou do controle dos graus de liberdade(31).

Com este quadro apresentado, uma das possibilidades de se avaliar a assimetria entre os lados é a análise da variabilidade de movimento durante a realização do chute ${ }^{(18,22,28)}$. Com isso, é possível a intervenção mais detalhada durante o aprendizado e o treinamento para diminuir a distinção encontrada entre os lados e melhorar o controle motor do atleta durante o movimento de chute, o que para o jogador de futsal é importante para o alcance de alto nível de destreza esportiva.

Diante do exposto, o objectivo do presente estudo foi analisar o desempenho e a variabilidade do movimento das articulações do quadril, joelho e tornozelo do membro de suporte do chute realizado com o MD e MND no futsal.

\section{MATERIAS E MÉTODOS}

\section{Participantes}

Participaram deste estudo 12 jogadores de futsal com idade entre 13 e 14 anos $(57,9 \pm 12,9 \mathrm{~kg} ; 1,62 \pm 0,06$ $\mathrm{m})$. Estes foram informados dos procedimentos e os seus responsáveis concederam a autorização via termo de consentimento livre e esclarecido aprovado pelo comité de ética da instituição. Todos os participantes eram destros para o membro de chute e, consequentemente, sinistros para o membro de suporte. Esta configuração foi observada nas acções dos atletas em treinamentos e partidas de futsal.

\section{Tarefa}

A tarefa dos participantes consistiu em realizar, após um apito, cinco chutes com o dorso do pé com cada membro (MD e MND). A bola estava parada a $10 \mathrm{~m}$ do gol (tiro livre do futsal). Foi pedido aos partici- 
pantes que realizassem os chutes com máxima velocidade da bola e tentassem acertar um alvo de $1 \mathrm{~m}^{2}$ posicionado no centro do gol. Os chutes foram precedidos por um aquecimento para evitar contusões e realizados em uma quadra oficial de futsal para se aproximar de uma situação real de jogo. A bola utilizada seguiu o padrão definido pela FIFA para esta idade. Foi permitido aos participantes realizarem a corrida de aproximação da maneira preferida. A ordem dos chutes foi definida aleatoriamente.

\section{Procedimentos experimentais}

Os movimentos dos participantes foram filmados por quatro câmaras de vídeo digitais (JVC GRDVL9800u ${ }^{\circledR}$ ) ajustadas a uma frequência de aquisição de imagens $120 \mathrm{~Hz}$, e foco definido e fixado manualmente. As câmaras, sobre tripés, permaneceram posicionadas para que focalizassem os marcadores passivos (esferas de plástico brancas com $3,5 \mathrm{~cm}$ de diâmetro), que foram fixados externamente nas seguintes proeminências ósseas de ambos os membros inferiores dos participantes: trocânter maior do fémur, cabeça da tíbia e maléolo lateral. Para a calibração, utilizou-se um objecto em forma de paralelepípedo rectângulo com 16 marcadores com posições previamente mensuradas, que foi posicionado no local onde os participantes realizaram os movimentos (Figura 1).

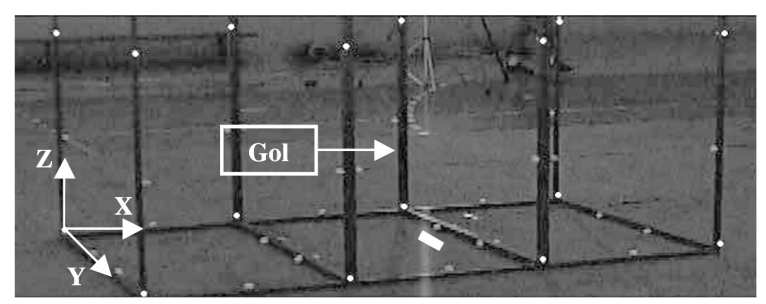

Figura 1. Objecto calibrador e sistema de referência definido.

Como forma de padronização do estudo, o ciclo de movimento analisado foi o mesmo para todos os participantes, tendo início na retirada do pé de chute do solo até a perda do contacto do pé de chute com a bola ( $100 \%$ do ciclo). O ciclo de movimento analisado foi ainda dividido em duas fases: fase de apoio (FA), com início na retirada do pé de chute do solo e fim no total aplainamento do pé de apoio no solo (65\% do ciclo), e fase de contacto (FC), com início no total aplainamento do pé de apoio no solo e fim no contacto do pé de chute com a bola $(35 \%$ do ciclo) (Figura 2).

\section{Obtenção das variáveis cinemáticas}

As imagens foram capturadas para um computador através da placa Studio DV da Pinnacle ${ }^{\circledR}$, para que fossem realizados os procedimentos de sincronização, medição, calibração e reconstrução tridimensional dos marcadores através do software de videogrametria "Dvideow", Digital Video for Biomechanics for Windows 32 bits $^{(6,11)}$. Dois sinais sonoros (apitos) foram utilizados para a sincronização das imagens, um emitido anterior a execução do chute e outro emitido após o contacto do pé com a bola. A medição dos marcadores ocorreu através do tracking automático, sendo realizadas correcções manuais quando necessário. A obtenção de coordenadas espaciais dos marcadores a partir de suas projecções em imagens é denominada reconstrução tridimensional de coordenadas e, neste caso, foi utilizado o método DLT - Direct Linear Transformation ${ }^{(1)}$, sendo necessária a filmagem simultânea de cada ponto por no mínimo duas câmaras.

\section{Tratamento dos dados}

Para o tratamento dos dados foi utilizado o programa MATLAB $6.5^{\circledR}$. Os dados obtidos da reconstrução tridimensional foram suavizados para separar o sinal do ruído, através da função não paramétrica ponderada local robusta Loess ${ }^{(10)}$. Esta função mostra-se adequada a este tipo de análise por ter um ajuste não paramétrico, ou seja, não leva em conta a existência de um modelo para este tipo de movimento, fazendo assim com que a sua forma seja referente ao conjunto dos dados apresentados.

Além disso, também foi realizado um teste para determinar a acurácia do estudo, denominação utilizada para avaliar o erro do experimento. Um estudo muito acurado apresenta um elevado grau de concordância entre o resultado obtido e o fenómeno estudado(34). Sua determinação foi feita considerando os valores de erros sistemáticos (bias) e aleatórios (precisão). Para isso, foi filmada a movimentação de forma aleatória de uma haste rígida, com dois marcadores passivos de $3,5 \mathrm{~cm}$ de diâmetro fixados um 


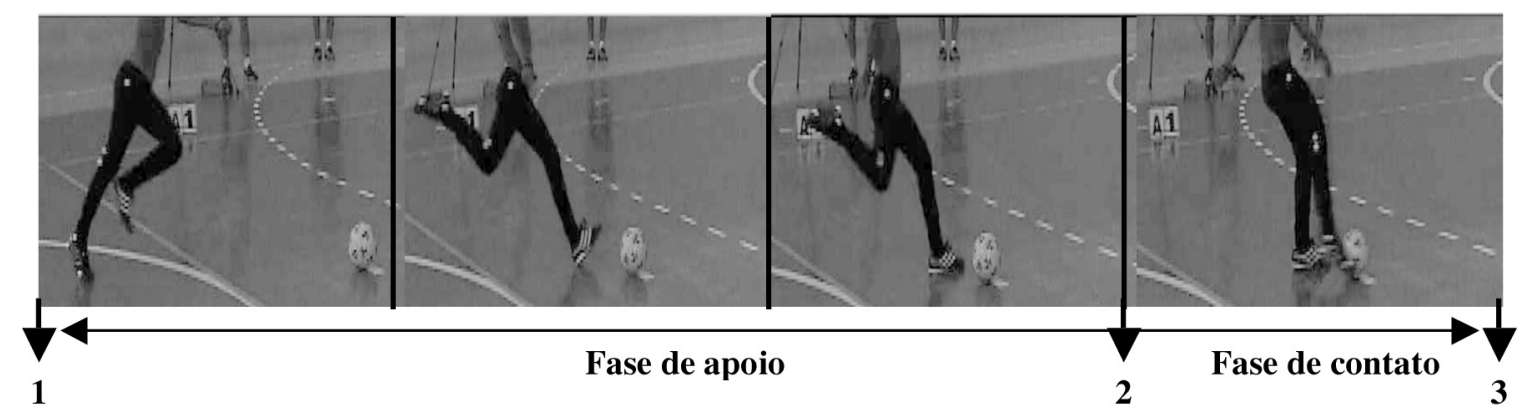

Figura 2. Retirada do pé de chute do solo [1], total aplainamento do pé de apoio no solo (2) e toque do pé de chute com a bola [3], definindo as fases de apoio e contacto.

em cada extremidade, por toda a região onde o objecto de calibração foi posicionado. A distância entre os dois marcadores foi obtida dez vezes utilizando uma trena com escala em milímetros (medição directa). Então, a média destas medidas (valor real) foi calculada. As imagens deste objecto passaram pelos processos para obtenção das variáveis cinemáticas descritos. A distância Euclidiana entre os dois marcadores para cada instante de tempo foi então calculada, sendo este valor adoptado como o valor mensurado. Assim, os valores do erro sistemático (exactidão ou bias) e do erro aleatório (precisão) para a verificação da acurácia (equações 1,2 e 3 ) foram calculados. Deste modo, quanto menor for o valor da acurácia mais acurados foram os dados obtidos no estudo.

$$
p=\sqrt{\frac{1}{n-1} \sum_{i=1}^{n}(d(i)-\bar{m})^{2}}
$$

(1)

$$
\mathbf{b}=\frac{1}{\mathbf{n}} \sum_{\mathbf{i}=\mathbf{1}}^{\mathbf{n}}|\grave{l}-\mathbf{d}(\mathbf{i})|
$$

(2)

$$
a=\sqrt{b^{2}+p^{2}}
$$

(3)

Nas equações acima: $d[i]$ é a distância Euclidiana entre os dois marcadores; $i=1, \ldots$, n é o número de medidas realizadas no cálculo da distância entre os dois marcadores (número de linhas da matriz de dados); $\bar{m}=$ valor médio das $n$ medidas; $\mu$ é o valor real da distância entre os dois marcadores por medição directa; $p$ é a precisão (1); b é o bias [2]; a é a acurácia (3).

\section{Desempenho}

Os desempenhos nos chutes foram analisados através da observação do acerto ou erro no alvo. Para isso, foram anotados após cada chute o local de acerto da bola.

\section{Cálculo da variabilidade do membro de suporte}

Após a suavização dos dados, os movimentos ocorridos nas articulações do quadril, joelho e tornozelo foram representados pelos marcadores do trocânter maior do fémur, cabeça da fíbula e maléolo lateral respectivamente. A variabilidade do movimento emerge dos múltiplos graus de liberdade inerentes no sistema motor(7). Quando se realiza uma mesma tarefa várias vezes, tendo o mesmo objectivo, por exemplo, acertar o centro do alvo no arco e flecha, a pequena variabilidade do movimento é de extrema importância para que se tenha um óptimo rendimento. Desta maneira, para o caso do movimento de chute quando se tem sempre o mesmo objectivo (acertar o alvo), quanto mais próximo for um movimento em relação ao outro, menos variável ele será. No entanto, deve-se ter cuidado, pois o movimento pode apresentar pequena variabilidade e bom desempenho, quando se acerta o alvo (movimento preciso e exacto), mas também pode apresentar pequena variabilidade e mau desempenho, quando não se acerta o alvo (movimento preciso e não exacto) (28). A variabilidade associada de cada articulação foi avaliada através do autovalor(16) da matriz de covariância dos movimentos das articulações do MD e MND. Para isso, foi calculado, para cada instante de tempo, 
o autovalor dos dados das articulações em questão para o MD e MND. O autovalor obtido corresponde à variabilidade da matriz de dados referente a cada articulação e o espalhamento dos pontos foi calculado através da raiz quadrada dos valores que correspondem ao desvio padrão dos dados(16). Desta forma, consegue-se representar a variabilidade dos movimentos das articulações nos chutes.

\section{Análise estatística da variabilidade}

Para determinar se existem diferenças na variabilidade do movimento entre os segmentos de suporte dominante e não dominante foi calculado um coeficiente de variabilidade. Para isso, utilizou-se o software MATLAB $6.5^{\circledR}$. Assim, foi calculada, em cada instante de tempo, a razão do espalhamento dos autovalores entre as respectivas articulações do quadril, joelho e tornozelo do MD e MND. Para verificar se houve diferenças entre os lados foi utilizada a função de distribuição acumulada(35). Os valores desta função que estiverem acima de 0,95 $(\mathrm{p}<0,05)$ foram considerados significantemente diferentes, ou seja, existe diferença entre as execuções em função de uma maior variabilidade de movimento de uma articulação de um lado em relação à mesma articulação do outro lado. Também foi verificada a diferença entre as articulações intramembro através da análise de variância com factor para a fase de chute e articulação $(p<0,05)$ do valor médio dos autovalores das articulações MD e MND. Para verificar a relação entre estas articulações, para cada fase de chute, a correlação de Pearson foi empregada. O desempenho foi analisado pela percentagem de acerto e erro no alvo. As análises estatísticas foram executadas no programa SPSS $10.0^{\circledR}$ (SPSS, Inc.).

\section{RESULTADOS}

A acurácia verificada no estudo foi de $1,42 \mathrm{~cm}$, de tal forma que o valor da precisão encontrado foi 1,05 $\mathrm{cm}$ e para o bias de $0,50 \mathrm{~cm}$. Esta medida pode ser considerada pequena, pois não excede a medida do raio do marcador utilizado.

Os resultados mostraram melhor desempenho para o lado dominante em relação ao não dominante (Figura 3). Os chutes com o MD apresentaram mais acertos do alvo $(36,7 \%)$ que o MND (5\%).

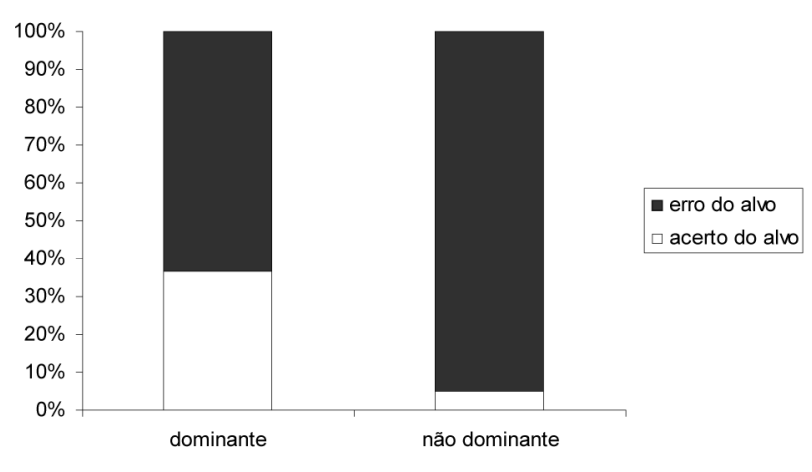

Figura 3. Desempenho dos chutes com o MD e MND.

A Tabela 1 apresenta as médias e desvios padrão dos autovalores das articulações dominantes e não dominantes. Observa-se que o movimento no membro de suporte apresentou maior variabilidade na articulação proximal (quadril) do que nas articulações distais (joelho e tornozelo) em ambos os lados. Do mesmo modo, foi verificada alta correlação significativa $(p<0,05)$ entre as variabilidades das articulações do lado não dominante na fase apoio (quadril e joelho: $r=0,93$; quadril e tornozelo: $r=0,88$; joelho $\mathrm{e}$ tornozelo: $r=0,84$ ), mostrando que as articulações têm alta correlação para a variabilidade. $\mathrm{Na}$ fase de contacto, encontrou-se correlação inversa entre a articulação do quadril com a do joelho $(r=-0,98)$ e do quadril com a do tornozelo $(r=-0,71)$. Para o lado dominante, na fase de apoio, só foi encontrada correlação entre variabilidade de joelho e tornozelo $(r=0,78)$ e, na fase de contacto, entre as articulações do quadril e do joelho $(r=0,93)$.

As variabilidades das articulações do quadril, joelho e tornozelo do membro de suporte dominante e não dominante estão representadas na Figura 4. O quadril não apresentou diferença entre os lados durante todo o ciclo de movimento, não havendo assimetria para esta articulação $(\mathrm{p}<0,05)$. Já para a articulação do joelho, verificou-se assimetria entre os membros inferiores durante toda a fase de apoio e parte da fase de contacto, até aproximadamente $75 \%$ do ciclo do chute $(p<0,05)$. A articulação do tornozelo apresentou assimetria entre os lados durante a fase de apoio, até $52 \%$ do ciclo de movimento $(p<0,05)$, sendo que na fase de contacto não houve diferença na variabilidade. Nos locais onde apareceram diferenças sempre foi encontrada maior variabilidade para MND (Tabela 1). 
Tabela 1. Médias e desvios padrão dos autovalores (em metros] das articulações dominantes e não dominantes.

Membro dominante Membro não dominante

\begin{tabular}{|c|c|c|c|c|c|}
\hline \multicolumn{6}{|c|}{ FASE DE APOIO } \\
\hline Quadril* & Joelho** & Tornozelo & Quadril * & Joelho** & Tornozelo \\
\hline $0,28 \pm 0,01$ & $0,19 \pm 0,006$ & $0,12 \pm 0,03$ & $0,37 \pm 0,01$ & $0,41 \pm 0,02$ & $0,24 \pm 0,03$ \\
\hline \multicolumn{6}{|c|}{ FASE DE CONTACTO } \\
\hline Quadril * & Joelho** & Tornozelo & Quadril* & Joelho** & Tornozelo \\
\hline $0,28 \pm 0,01$ & $0,23 \pm 0,009$ & $0,16 \pm 0,003$ & $0,36 \pm 0,003$ & $0,35 \pm 0,005$ & $0,21 \pm 0,0003$ \\
\hline
\end{tabular}

* quadril diferente de joelho e tornozelo; ${ }^{* *}$ joelho diferente de tornozelo $(p<0,001)$.
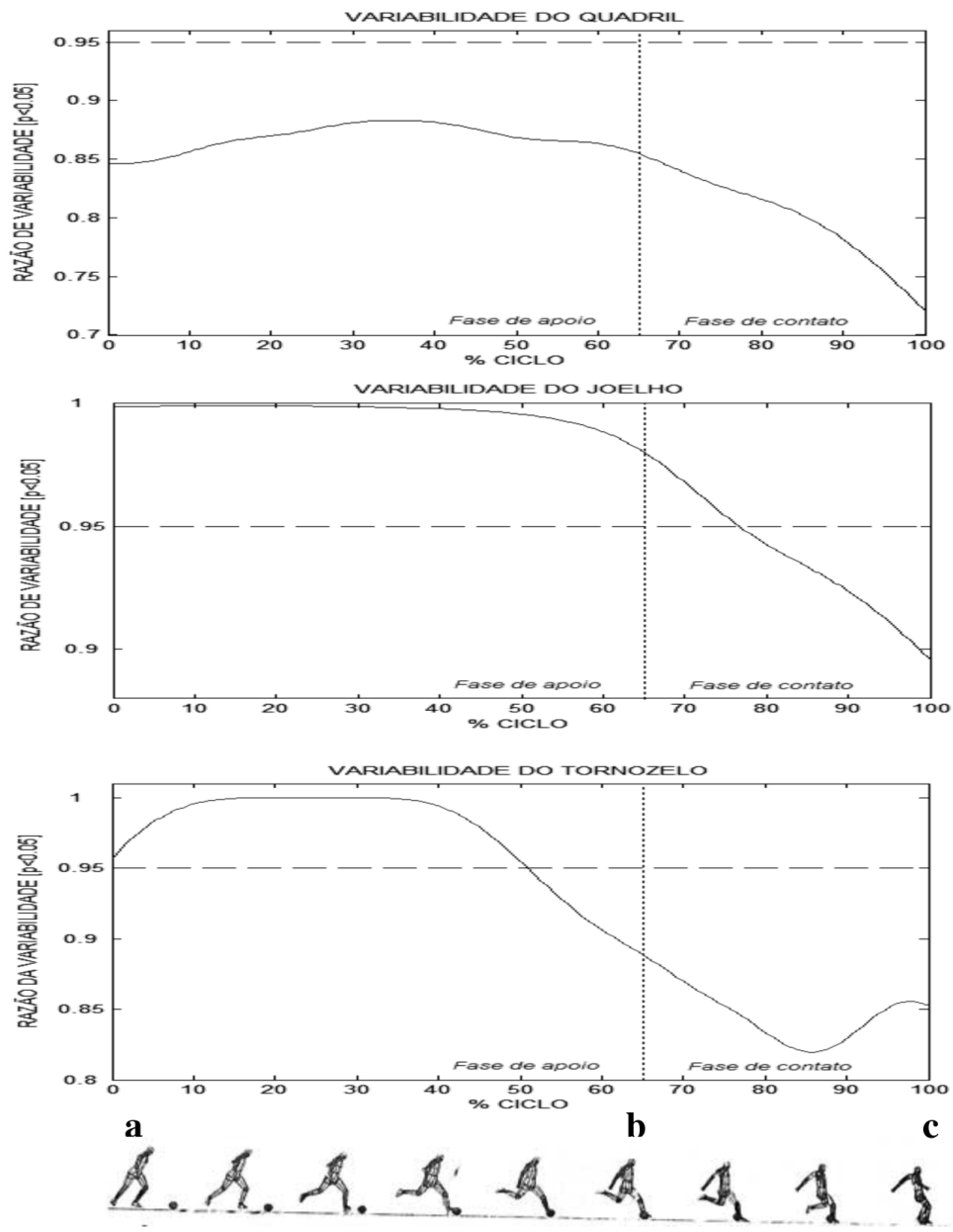

Figura 4. Coeficiente de variabilidade da articulação do quadril, joelho e tornozelo. a) o último contato do membro de chute no solo. b) total aplainamento do pé de suporte no solo. c) primeiro contato do membro de chute com a bola. 


\section{DISCUSSÃO}

A ferramenta utilizada no estudo se apresentou eficaz para análise da variabilidade. Por meio dos conceitos matemáticos, que às vezes são de difícil interpretação, apresentou-se o que ocorreu na variabilidade de ambos os membros durante o movimento de chute. Como esperado, a variável desempenho mostrou-se diferente entre os lados. O MD mostrou desempenho maior (37,4\% de acerto no alvo) em relação ao MND (5\% de acerto no alvo) (Figura 3). Estes resultados corroboram com os resultados de outros estudos, que também encontraram pior desempenho com o MND, variando entre $20 \%$ e $50 \%(3,15,19,32)$. $\mathrm{Na}$ análise da variabilidade intra-membros para o lado dominante e não dominante, verificou-se maior variabilidade da articulação proximal (quadril) em relação às articulações distais (joelho e tornozelo) tanto na fase de apoio quanto na fase de contato (Tabela 1). A articulação do quadril é a que mais oscila durante o movimento de chute e a partir dela surgem as correcções/ajustes no movimento. Isto acontece porque além desta articulação ter maior possibilidade de movimento comparado com as articulações do joelho e tornozelo, os ajustes na articulação proximal reflectem nas articulações distais, facilitando o controle do movimento. A maior mobilidade da articulação do quadril está relacionada ao maior número de graus de liberdade dessa articulação em relação às outras. Além disso, o movimento dessa articulação colabora para o movimento de alavanca do membro de chute, revelando sua importância para o membro de suporte para ambos os lados. Para o lado não dominante, especificamente, foi encontrada alta correlação significativa na variabilidade das articulações, demonstrando que ajustes estavam sendo executados. Na fase de apoio, as articulações do MND estão positivamente correlacionadas entre si, ou seja, há aumento da variabilidade em todas as articulações. Na fase de contacto, as articulações do joelho e tornozelo não apresentam correlação entre si, mas estão inversamente relacionadas com a articulação do quadril do MD, indicando que o sistema de controle está usando a articulação proximal para regular a variabilidade das articulações distais, diferentemente do membro de chute ${ }^{(25)}$. Dois pontos importantes devem ser destacados: 1) a exploração do MND: o controle do lado não dominante pode ser entendido como menos refinado(4); 2) a especificidade das fases do movimento: na fase de contacto com a bola há necessidade de diminuição da variabilidade, considerando especialmente que a meta a ser alcançada exige controle mais refinado(5), o que pode não ser necessário na fase de apoio.

Em relação ao MD, nenhuma correlação significativa foi encontrada. Este membro está sob um controle mais refinado, cada articulação pode ser individualmente controlada sem que haja aumento na variabilidade das articulações relacionada a ela, independente da fase do movimento.

Além disso, é consistente o achado das diferenças entre os lados centradas principalmente na fase de apoio, já que nesta fase o pé do membro de suporte apresenta um período de contacto com o solo bem curto (Figura 4). Após o contacto torna-se difícil a alteração do movimento, uma vez que existe a redução nos graus de liberdade do movimento(7), sendo necessário que o atleta realize alterações na acção anterior a este momento para que assim se atinja um melhor desempenho. As articulações do joelho e do tornozelo dominantes apresentaram, em alguns momentos do ciclo de chute, menor variabilidade do que as respectivas articulações não dominantes, principalmente na fase de apoio. O MND apresenta menor habilidade e pobre coordenação se comparado ao $\mathrm{MD}^{(4,5)}$, concordando com os achados deste estudo. Sob esta perspectiva, a menor variabilidade do movimento do MD é reflexo de um programa motor sofisticado, onde o sistema é mais preciso ${ }^{(22)}$. O MND apresenta movimentos imaturos e pouco repetitivos, representado pela maior variabilidade, como verificado em jogadores amadores ${ }^{(27)}$. Ao contrário, o MD se encontra em um estágio mais maduro de movimento, representado pela maior consistência de movimento.

A maior variabilidade do MND sustenta os estágios de controle propostos por Bernstein(7) e Newell(20). Desta forma, como o MND está em uma fase motora de aprendizado e refinamento, os jogadores procuram aprimorar seu movimento durante as tentativas e, consequentemente, o desempenho na tarefa. Com isso, observa-se maior variabilidade de movimento e baixo desempenho. Já para o lado dominante, que é 
o mais usado e se apresenta com alto grau de destre$\mathrm{za}$, os jogadores procuram repetir os movimentos anteriores a fim de atingir o objectivo proposto na tarefa. Desta forma, o MD apresenta maior consistência de movimento, que é a "marca registada" de um movimento de alto nível( $(9,33)$, especialmente em tarefas de natureza fechada(28).

Um facto agravante para o aumento na assimetria dos movimentos contralaterais é que as pessoas destras utilizam o membro esquerdo para o suporte durante as acções cotidianas ${ }^{(14)}$, o que é conhecido como assimetria funcional e reforça a distinção dos movimentos. No entanto, estas assimetrias na variabilidade do movimento e no desempenho podem ser reduzidas ou sanadas $(15,32)$. Ponderando que o grupo de participantes do presente estudo é composto por atletas que não podem, ainda, ser considerados como de alto nível, é possível reduzir a variabilidade das articulações para melhorar o desempenho. Esta informação se faz útil aos treinadores de futsal, que devem propiciar ao praticante maior número de acções com o MND, fazendo com que realizem mais chutes e movimentos e, assim, melhorem a capacidade técnica e a coordenação. Com uma intervenção no movimento dos jogadores, ambos os membros poderão ter acções habilidosas que são marcadas pela pouca variabilidade do movimento ${ }^{(9,31)}$. Para que este fato se efective, deve ser dada ênfase similar para os dois membros inferiores durante o aprendizado do futsal, pois se a importância for distinta poderá evidenciar, ainda mais, a assimetria do movimento. $\mathrm{O}$ alto desempenho do atleta com ambos os membros facilita as acções durante a partida, pois aumenta as possibilidades de acções tácticas e técnicas. Além disso, para um desempenho de sucesso nos chutes, o atleta necessita apresentar bom equilíbrio e controle postural(29), que também serão beneficiados com o aumento do treinamento com o MND.

Correlacionando o desempenho e a variabilidade, encontra-se que a menor variabilidade do movimento encontrada do lado dominante auxilia no alto desempenho para este lado, ocorrendo o contrário para o membro contralateral. De acordo com Tani(31), quando se realiza um movimento habilidoso procura-se adoptar uma estratégia que promova relativa invariância do movimento mesmo com alta exigência da tarefa.

\section{CONCLUSÃO}

Conclui-se que as articulações do joelho e tornozelo do membro de suporte não dominante analisadas neste estudo tiveram maior variabilidade de movimento do que as respectivas articulações do MD, que apresentaram maior consistência de movimento, principalmente na fase de apoio. A articulação do quadril dirige o MND, tanto na fase de apoio como na de contacto. Além disso, o MD apresentou melhor desempenho provavelmente causado pela menor variabilidade do movimento. Há assimetria entre os membros contralaterais de suporte, influenciando o desempenho do chute no futsal e demonstrando a importância do membro de suporte para o chute.

\section{AGRADECIMENTOS}

Os autores agradecem o apoio da FAPESP (00/07258-3), FUNDUNESP, PIBIC/CNPq e FAPEMIG para a realização deste trabalho.

\section{CORRESPONDÊNCIA}

\section{Lilian Teresa Bucken Gobbi}

Laboratório de Estudos da Postura e da Locomoção Departamento de Educação Física - IB/UNESP/Rio Claro

Av. 24-A, 1515 - Bela Vista - CEP: 13.506-900 - Rio Claro/São Paulo/Brasil

Fone/Fax: + 5519 3534-6436

E-mail: ltbgobbi@rc.unesp.br 


\section{REFERÊNCIAS}

1. Abdel-Aziz YI, Karara HM (1971). Direct linear transformation from comparator coordinates into object-space coordinates. Proc. ASP/UI Symp. On Close-range Photogrammetry (Urbana, 1995). American Society of Photogrammetry, Falls Church (Va/USA), 1-18.

2. Asami T, Nolte V (1983). Analysis of powerful ball kicking. In: Matsui H, Kobayashi K (ed.) Biomechanics VIII-B, Champaign: Human Kinetics, 695-700.

3. Barbieri FA, Gobbi LTB, Lima Junior RS (2006). Aspectos da corrida de aproximação entre o chute realizado com o membro dominante e não dominante. Rev Motricidade 2: 80-90.

4. Barfield WR (1995). Effects of selected kinematics and kinetic variables on instep kicking with dominant and nondominant limbs. J Hum Mov Stud 29: 251-272.

5. Barfield WR, Kirkendall DT, Yu B (2002). Kinematic instep kicking differences between elite female and male soccer players. J Sports Sci Med 1: 72-79.

6. Barros RML, Brezikofer R, Leite NJ, Figueroa PJ (1999). Desenvolvimento e avaliação de um sistema para análise tridimensional de movimentos humanos. Rev Bras Eng Bioméd 15: 79-86.

7. Bernstein N (1967). The coordination and regulation of movements. Oxford: Pergamon.

8. Carey DP, Smith G, Smith DT, Shepherd JW, Skriver J, Ord L, Rutland A (2001). Footedness in world soccer: an analysis of France '98. J Sports Sci 19: 855-864.

9. Connolly KJ (1977). The nature of motor skill development. J Hum Mov Stud 3: 128-143.

10. Cunha AS, Lima Filho EC (2003). Metodologia para a suavização de dados biomecânicos por funções não paramétricas. Braz J Biomec 1: 23 - 28.

11. Figueroa PJ, Leite NJ, Barros RML (2003). A flexible Software for tracking of markers used in Human Motion Analysis. Comp Meth Prog Biomed 72: 155-65.

12. Gentry V, Gabbard C (1995). Foot-preference behavior: a developmental perspective. J Gen Psychol 122: 37-45.

13. Gobbi LTB, Secco CR, Marins FHP (2001). Preferência pedal: comportamento locomotor em terreno irregular. In: Teixeira LA (ed.) Lateralidade e Comportamento Motor: assimetrias laterais de desempenho e transferência interlateral de aprendizagem, São Paulo: Movimento, 225-247.

14. Goble DJ, Marino GW, Potvin JR (2003). The influence of horizontal velocity on interlimb symmetry in normal walking. Hum Mov Sci 22: 271-283.

15. Haaland E, Hoff J (2003). Non-dominant leg training improves the bilateral motor performance of soccer players. Scand J Med Sci Sports 13: 179-184.

16. Harville DA (1997). Matrix algebra from a statistician's perspective. New York: Springer Science, 515-582.

17. Isokawa M, Lees A (1988). A Biomechanical analysis of the instep kick motion in soccer. In: Reilly T (ed.). Science and Football. London: Routledge, 449-455.

18. Lees A, Nolan L (2002). Three dimensional kinematic analyses of the instep kick under speed and accuracy conditions. In: Reilly T (ed.). Science and Football IV, Sydney: E\&FN Spon, 22-26.

19. McLean BD, Tumilty DMcA (1993). Left-right asymmetry in two types of soccer kick. Br J Sports Med 27: 260-262.

20. Newell KM (1986). Change in movement and skill: Learning, retention and transfer. In: Wade MG, Whiting
HTA (ed.). Motor development in children: Aspects of coordination and control. Dordrecht: Martinus Nijhoff, 341-359.

21. Nunome H, IkegamI Y, Kozakai R, Apriantono T, Sano S (2006). Segmental dynamics of soccer instep kicking with the preferred and non-preferred leg. J Sports Sci 24: 529540.

22. Phillips SJ (1985). Invariance of elite kicking performance. In: Winter D, Biomechanics $I X-B$, Champaign: Human Kinetics, 539-542.

23. Peters M (1988). Footedness: asymmetries in foot preference and skill and neuropsychological assessment of foot movement. Psychol Bull 103: 179-192.

24. Porac C, Coren S (1981). Lateral preferences and human behavior. New York: Springer-Verlag.

25. Putnam CA (1991). A segment interacion analysis of proximal-to-distal sequential segment motion patterns. Med Sci Sports Exerc 23: 130-144.

26. Sadeghi H, Allard P, Prince F, Labelle H S (2000). Symmetric and limb in able-bodied gait: a review. Gait Posture 12: 34-45.

27. Saggini R, Calligaris A, Montanari G, Tjouroudis N, Vecchiet L (1993). The foot-ground reaction in the soccer player. In: Reilly T (ed.). Science and Football II. London: E\&FN Spon, 341-344.

28. Schmidt RA, Wrisberg CA (2001). Aprendizagem e performance motora. Rio de Janeiro: Artmed.

29. Sforza C, Dunani S, Mauro F, Torri L, Ferrario VF (1997). Repeatability of the football penalty: a statical evaluation by the morphological variation analysis. In: Reilly $\mathrm{T}$ (ed.). Science and Football III. London: E\&FN Spon, 240-245.

30. Starosta W (1993). Symmetry and asymmetry in shooting demonstrated by elite soccer players. Science and Football II. London: E\&FN Spon, 346-355.

31. Tani G (2000). Variabilidade e programação motora. In: Barbanti (ed.) A biodinâmica do movimento humano e suas relações interdisciplinares, 245-260.

32. Teixeira LA, Silva MVM, Carvalho MA (2003). Reduction of lateral asymmetries in dribbling: the role of bilateral practice. Laterality 8: 53-65.

33. Turvey MT (1977). Preliminaries to theory of action with to vision. In: Shaw R, Brandford J (ed.). Perceiving, acting and knowing: toward an ecological psychology. Nova Jersey: Lawrence Erbaum.

34. Vuolo JH (1996). Fundamentos da Teoria de Erros. São Paulo: Edgard Blücher.

35. Whitmore GA (1993). Applied Statistics. Boston: Allyn and Bacom. 